'Instituto de Farmacia, Facultad de Ciencias.

${ }^{2}$ Instituto de Anatomía, Histología y Patología, Facultad de Medicina. ${ }^{3}$ Instituto de Salud Pública, Facultad de Medicina ${ }^{4}$ Instituto de Enfermería, Facultad de Medicina.

Instituto de Bioquímica, Facultad de Ciencias.

${ }^{6}$ Escuela de Química y Farmacia Facultad de Ciencias. Universidad Austral de Chile Valdivia, Chile.

aBioquímico, Mg.

bProfesor de Biología, Química y Ciencias, Mg.

Médico Veterinario, Mg.

'Enfermera, Mg eBioquímico, Mg.

fEstudiante tesista Escuela de Química y Farmacia.

Proyecto interno S-2007-29 "Factores de Riesgo cardiovascular en Estudiantes de la Universidad Austral de Chile", financiado por la Dirección de Investigación y Desarrollo de la Universidad Austral de Chile.

Recibido el 25 de abril de 2011 aceptado el 20 de diciembre de 2011.

Correspondencia a Ma Adela Martínez S. Bioquímico, Mg. Instituto de Farmacia, Facultad de Ciencias, Universidad Austral de Chile, Valdivia, Chile. Casilla 567 Valdivia, Chile. E-mail:mmartin3@uach.cl

\section{Factores de riesgo cardiovascular en estudiantes de la Universidad Austral de Chile}

\author{
MARÍA ADELA MARTÍNEZ S., ${ }^{1, a}$, ANA MARÍA LEIVA O.,b, \\ CRISTINA SOTOMAYOR C., , , TATIANA VICTORIANO R., ,, \\ ANA MARÍA VON CHRISMAR P.,e, SANDRA PINEDA B. ${ }^{6, \mathrm{f}}$

\section{Cardiovascular risk factors among university students}

Background: Cardiovascular diseases are related to particular lifestyle patterns and the presence of cardiovascular risk factors (CVRF). Aim: To evaluate the presence of CVRF in students from Universidad Austral de Chile (UACh). Material and Methods: CVRF were evaluated in 385 university students aged 17 to 26 years (63\% women). Personal background, lifestyle, anthropometry, blood pressure, serum lipids and blood glucose were evaluated. Results: Eighty eight percent of evaluated students had sedentary habits, 19\% had high LDL cholesterol levels, $40 \%$ had high blood pressure, $28 \%$ smoked, 29\% were overweight or obese and 20\% had some stress level. Conclusions: There is a high frequency of non-healthy lifestyles and cardiovascular risk factors in this sample of university students.

(Rev Med Chile 2012; 140: 426-435).

Key words: Cardiovascular diseases; Risk factors; Students.
L as enfermedades cardiovasculares (ECV) constituyen un problema de salud pública al ser la principal causa de muerte e incapacidad a nivel mundial ${ }^{1}$; son de etiología múltiple y su evolución está ligada fundamentalmente a la presencia de factores de riesgo cardiovascular (FRCV), los cuales al ser en su mayoría susceptibles de ser modificados, se transforman en la clave de su prevención ${ }^{2}$.

Chile, al igual que la mayoría de los países del mundo, ha experimentado cambios negativos en los estilos de vida de su población, lo que ha contribuido a una mayor prevalencia de FRCV modificables ${ }^{3}$, siendo las ECV la causa más frecuente de mortalidad $(27 \%)^{4}$.

Diversos estudios en Chile evidencian un incremento de estos FRCV en los jóvenes, destacándose una elevada prevalencia de sedentarismo, tabaquismo, sobrepeso u obesidad e hipercolesterolemia, entre otros ${ }^{3,5-8}$. Lo anterior, nos lleva a suponer que los estudiantes que ingresan a la Universidad Austral de Chile (UACh), no estarían ajenos a esta realidad.

El objetivo del presente trabajo es evaluar la presencia de FRCV en estudiantes de la UACh, con el fin de conocer la realidad local, y si es pertinente, a futuro, diseñar estrategias de promoción de estilos de vida saludable en la comunidad universitaria.

\section{Material y Método}

Población y diseño del estudio

Se realizó un estudio descriptivo de corte transversal, con análisis de FRCV. La muestra quedó conformada por 385 estudiantes de un universo de 1.557 , que ingresaron al primer año de las carreras de pregrado de la UACh, en Valdivia el año 2007. El tamaño muestral, para estimar proporciones, se calculó asumiendo un nivel de confianza de 
Factores de riesgo cardiovascular en estudiantes universitarios - M. A. Martínez et al

95\%, una frecuencia esperada de eventos a evaluar de $50 \%$ y un error estimado de $5 \%(p=0,05)$. El muestreo fue en dos etapas; primero se estratificó por carrera profesional y posteriormente se aplicó un muestreo aleatorio sistemático $(\mathrm{k}=4)$. Se invitó a participar en el estudio a los estudiantes seleccionados, reemplazándose a los que no accedieron.

La información socioeconómica fue proporcionada por la Dirección de Asuntos Estudiantiles (DAE) de la UACh, que clasifica a los estudiantes en quintiles de ingreso per-cápita del hogar, de acuerdo a los datos aportados por la Encuesta de Caracterización Socioeconómica Nacional (CASEN). El quintil I corresponde a estudiantes pertenecientes a hogares con ingreso mensual hasta $\$ 39.678$ (CLP) por integrante del grupo familiar; quintil II: estudiantes de hogares con ingresos mensuales entre $\$ 39.678$ y $\$ 67.658$ per-cápita; quintil III: estudiantes de hogares con ingresos mensuales entre $\$ 67.658$ y $\$ 106.867$ per-cápita; quintil IV: estudiantes de hogares con ingresos mensuales entre \$106.867 y \$199.907 per-cápita y quintil V: estudiantes de hogares con ingresos mensuales desde \$199.907 per-cápita9 .

\section{Recolección de la información}

Se realizó durante agosto y septiembre de 2007, previa firma de consentimiento informado. Los estudiantes asistieron en ayuno de 12 horas, con el fin de extraer una muestra de sangre venosa, para determinar glicemia y perfil lipídico. Paralelamente, se tomaron medidas antropométricas, se midió la presión arterial y se aplicó un cuestionario.

\section{Cuestionario}

Se diseñó un cuestionario de auto-aplicación en base al instrumento de la II Encuesta Nacional de Calidad de Vida y Salud (ECV 2006) ${ }^{10}$ y el documento Herramienta para Vigilancia de Enfermedades No Transmisibles de la Organización Panamericana de la Salud ${ }^{11}$. Este fue previamente validado y consideró antecedentes personales, familiares, situaciones de la vida cotidiana y estilos de vida (estrés, actividad física, consumo de tabaco, consumo alcohol, entre otros).

\section{Mediciones antropométricas}

Para peso y la talla se utilizó una balanza calibrada a $0,1 \mathrm{~kg}$ con cartabón adosado graduado a $0,2 \mathrm{~cm}$ (Detecto $\left.{ }^{\oplus}, \mathrm{USA}\right)$. Para circunferencia de cintura, se utilizó una cinta métrica flexible, graduada en milímetros, según protocolos de la $\mathrm{OMS}^{12}$.

\section{Presión arterial}

Se utilizó el método indirecto de auscultación de la arteria radial con un estetoscopio y un esfigmomanómetro anaeroide (Lumiscope ${ }^{\circledast}$ ), de acuerdo a protocolo establecido ${ }^{13}$, efectuándose dos mediciones posteriormente promediadas; la primera fue con un descanso previo de 5 minutos y la segunda 5 minutos después.

\section{Exámenes de laboratorio}

Las muestras de sangre fueron procesadas en el Laboratorio de Bioquímica de la UACh, utilizándose métodos enzimáticos colorimétricos realizados en forma manual. Se determinó glicemia, colesterol total (CT), triglicéridos (TG) y colesterol HDL (cHDL), usando reactivos de Wiener Laboratorios S.A.I.C. ${ }^{\oplus}$ y espectrofotómetro doble

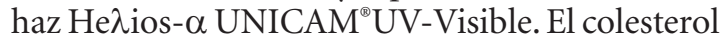
LDL (cLDL) se estimó utilizando la fórmula de Friedewald ${ }^{14}$.

Los puntos de corte para cada FRCV se definieron en base a los siguientes criterios:

Índice de masa corporal (IMC): Se consideró exceso de peso a un IMC $\geq 25 \mathrm{~kg} / \mathrm{m}^{2}$, sobrepeso a un IMC: $25-29,9 \mathrm{~kg} / \mathrm{m}^{2}$ y un IMC $\geq 30 \mathrm{~kg} / \mathrm{m}^{2}$ como obesidad ${ }^{3}$.

Circunferencia cintura (CC): Se consideraron 3 criterios para diagnosticar obesidad abdominal; criterio 1: "obesidad abdominal 1" a CC > $102 \mathrm{~cm}$ en varones, $\mathrm{CC}>88 \mathrm{~cm}$ en mujeres, criterio 2 : "obesidad abdominal 2" a CC $\geq 94 \mathrm{~cm}$ en varones, $\mathrm{CC} \geq 80 \mathrm{~cm}^{\mathrm{en}}$ mujeres ${ }^{3}$ y el recientemente validado para la población chilena, criterio 3: "obesidad abdominal 3" a CC $\geq 88 \mathrm{~cm}$ para varones, $C C \geq 83$ $\mathrm{cm}$ para mujeres ${ }^{8,15}$.

Perfil lipídico: Para el diagnóstico de las dislipidemias se consideró valores elevados de CT $\geq 200$ $\mathrm{mg} / \mathrm{dL}, \mathrm{cLDL} \geq 130 \mathrm{mg} / \mathrm{dL}, \mathrm{TG} \geq 150 \mathrm{mg} / \mathrm{dL}$ y valores bajos de $\mathrm{cHDL}<40 \mathrm{mg} / \mathrm{dL}^{16}$.

Glicemia: En condiciones de ayuno, se consideró glicemia elevada $\geq 100 \mathrm{mg} / \mathrm{dL}$ y posible diabetes no confirmada a valores de glicemia $\geq 126 \mathrm{mg} / \mathrm{dL}^{3}$.

Presión arterial (PA): Se consideró estado de pre-hipertensión, valores de PA sistólica (PAS) 120-139 mmHg o PA diastólica (PAD) 80-89 mmHg; y un estado de hipertensión arterial (HTA) valores de $\mathrm{PAS} \geq 140 \mathrm{mmHg}$ o $\mathrm{PAD} \geq 90 \mathrm{mmHg}^{17}$. 
Síndrome metabólico (SM): Para el diagnóstico del SM se utilizó el criterio propuesto por National Cholesterol Education Program Adult Treatment Panel III (NCEP ATP III) ${ }^{16}$, utilizando los puntos de corte para la población chilena para la obesidad abdominal $^{8,15}$.

Actividad física: Para medir actividad física se utilizó la pregunta del cuestionario aplicado en la ECV 2006, considerándose sedentario al estudiante que durante el último mes no practicó deporte o realizó actividad física fuera de su horario de trabajo, al menos tres veces por semana, durante 30 minutos $^{10}$.

Consumo de tabaco: Se consideró "fumador actual" al estudiante que señaló fumar, distinguiéndose entre ellos al "fumador diario": al menos un cigarrillo/día y "fumador ocasional": menos de un cigarrillo/día ${ }^{3}$ y "ex-fumador" al que había dejado de fumar.

Consumo de alcohol: Se evaluó la frecuencia de consumo durante la semana y la cantidad de tragos consumidos en un día habitual; considerándose bebedor moderado al que consume 1-2 tragos, bebedor en riesgo al que consume 3-5 tragos y bebedor excesivo si bebe más de 5 tragos en una sola ocasión ${ }^{18}$. Un trago estándar en Chile equivale en promedio a $15,5 \mathrm{~g}$ de alcohol absoluto ${ }^{8}$.

Estrés: Se consideró con estrés a aquellos estudiantes que manifestaron sentirse estresado siempre y casi siempre; y no estresados a aquellos que respondieron: algunas veces, rara vez o que nunca se sienten estresados ${ }^{10}$.

\section{Coexistencia de FRCV}

Se consideró seis FRCV clásicos: IMC $\geq 25$ $\mathrm{kg} / \mathrm{m}^{2}, \mathrm{PAS} \geq 120 \mathrm{mmHg}$ o $\mathrm{PAD} \geq 80 \mathrm{mmHg}$, CT $\geq 200 \mathrm{mg} / \mathrm{dl}$, glicemia $\geq 100 \mathrm{mg} / \mathrm{dL}$, tabaquismo y sedentarismo.

\section{Análisis de datos}

Se determinaron distribuciones absolutas y relativas para los parámetros evaluados. La distribución de las variables continuas se evaluó mediante la prueba de normalidad Shapiro-Wilk, asumiendo un nivel de significación de un 0,05. Se calcularon medidas resumen y de dispersión, además de las respectivas tasas de prevalencia e intervalos de confianza de los parámetros evaluados. La significancia estadística entre variables, género y quintil de ingreso, se realizó aplicando la prueba de $\chi^{2}(\mathrm{p}<0,05)$, mediante el programa estadístico SPSS 15.0.
Este estudio fue aprobado por el Comité de Ética de la UACh. Los resultados fueron confidenciales, derivándose al Centro Médico Estudiantil a los estudiantes con parámetros fuera de los rangos normales.

\section{Resultados}

\section{Caracterización de la muestra}

La muestra quedó constituida por 385 estudiantes de ambos géneros, rango de edad 17-26 años, $63,4 \%$ mujeres, edad promedio $18,8 \pm 1,0$ años y $36,6 \%$ varones, edad promedio $19,0 \pm 1,3$ años. Según información de ingreso autónomo per-cápita del hogar proporcionada por la DAE de la UACh, el 55,8\% de los estudiantes pertenecía a los tres quintiles de menor ingreso (Tabla 1).

\section{Índice de masa corporal}

El 28,6\% de los estudiantes presentaba exceso de peso, de los cuales $23,4 \%$ correspondió a sobrepeso y $5,2 \%$ a obesidad; sin diferencias significativas según género y quintil de ingreso (Tablas 2 y 3 ).

\section{Circunferencia cintura}

El 6\% de los estudiantes presentaba "obesidad abdominal 1", 23,9\% "obesidad abdominal 2" y 16,1\% "obesidad abdominal 3". La prevalencia de obesidad abdominal fue significativamente mayor en mujeres, para los tres criterios de diagnóstico utilizados; sin diferencias significativas según quintil de ingreso (Tablas 2 y 3 ).

\section{Perfil lipídico}

El 18,7\% de los estudiantes presentaba niveles de cLDL elevado y 5,7\% niveles de TG elevados; sin diferencias significativas entre género para

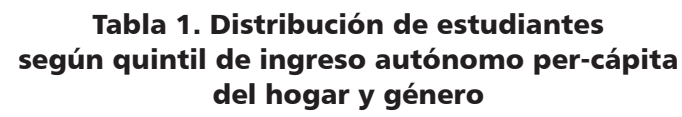

Tabla 1. Distribución de estudiantes según quintil de ingreso autónomo per-cápita del hogar y género

\begin{tabular}{|cccc|}
\hline Quintiles & $\begin{array}{c}\text { Mujeres } \\
\%\end{array}$ & $\begin{array}{c}\text { Varones } \\
\%\end{array}$ & $\begin{array}{c}\text { Total } \\
\%\end{array}$ \\
\hline I & 19,3 & 24,8 & 21,3 \\
\hline II & 16,0 & 16,3 & 16,1 \\
\hline III & 19,3 & 17,0 & 18,4 \\
\hline IV & 16,8 & 10,6 & 14,5 \\
\hline V & 28,7 & 31,2 & 29,6 \\
\hline
\end{tabular}

No hay significancia estadística p-valor $>0,05$. 
Tabla 2. Prevalencias e intervalos de confianza (95\%) de factores de riesgo cardiovascular en estudiantes de la Universidad Austral de Chile, según género

\begin{tabular}{|lrrrrrrr|}
\hline $\begin{array}{l}\text { Factor de riesgo } \\
\text { cardiovascular }\end{array}$ & \multicolumn{1}{c}{$\begin{array}{c}\text { Mujeres } \\
\text { \% }\end{array}$} & \multicolumn{2}{c}{$\begin{array}{c}\text { Varones } \\
\text { \% }\end{array}$} & \multicolumn{2}{c}{$\begin{array}{c}\text { Total } \\
\%\end{array}$} & p-valor \\
\hline Sobrepeso & 24,6 & $(19,0-30,2)$ & 21,3 & $(14,2-28,4)$ & 23,4 & $(19,0-27,7)$ & n.s. \\
\hline Obesidad & 5,3 & $(2,3-8,4)$ & 5,0 & $(1,0-8,9)$ & 5,2 & $(2,8-7,5)$ & n.s. \\
\hline Obesidad abdominal 1 & 8,2 & $(4,6-11,8)$ & 2,1 & $(0,4-6,1)$ & 6,0 & $(3,5-8,5)$ & $<0,05$ \\
\hline Obesidad abdominal 2 & 35,2 & $(29,0-41,4)$ & 4,3 & $(0,6-7,9)$ & 23,9 & $(19,5-28,3)$ & $<0,001$ \\
\hline Obesidad abdominal 3 & 20,9 & $(15,6-26,2)$ & 7,8 & $(3,0-12,6)$ & 16,1 & $(12,3-19,9)$ & $<0,001$ \\
\hline Colesterol total elevado & 18,4 & $(13,4-23,5)$ & 10,6 & $(5,2-16,1)$ & 15,6 & $(11,8-19,3)$ & $<0,05$ \\
\hline Colesterol LDL elevado & 20,9 & $(15,6-26,2)$ & 14,9 & $(8,7-21,1)$ & 18,7 & $(14,7-22,7)$ & n.s. \\
\hline Colesterol HDL bajo & 11,9 & $(7,6-16,2)$ & 22,7 & $(15,4-30,0)$ & 15,8 & $(12,1-19,6)$ & $<0,01$ \\
\hline Triglicéridos elevados & 6,1 & $(2,9-9,4)$ & 5,0 & $(1,0-8,9)$ & 5,7 & $(3,3-8,2)$ & n.s. \\
\hline Glicemia elevada & 2,0 & $(0,7-4,7)$ & 2,1 & $(0,4-6,1)$ & 2,1 & $(0,5-3,6)$ & n.s. \\
\hline Pre-hipertensión & 27,5 & $(21,7-33,3)$ & 48,2 & $(39,6-56,8)$ & 35,1 & $(30,2-40,0)$ & $<0,01$ \\
\hline Hipertensión & 2,0 & $(0,7-4,7)$ & 8,5 & $(3,6-13,5)$ & 4,4 & $(2,2-6,6)$ & $<0,01$ \\
\hline Síndrome metabólico & 4,9 & $(2,0-7,8)$ & 5,0 & $(1,0-8,9)$ & 4,9 & $(2,6-7,2)$ & n.s. \\
\hline Sedentarismo & 91,0 & $(87,2-94,8)$ & 82,3 & $(75,6-88,9)$ & 87,8 & $(84,4-91,2)$ & $<0,05$ \\
\hline Fumador actual & 28,7 & $(22,8-34,6)$ & 27,7 & $(19,9-35,4)$ & 28,3 & $(23,7-33,0)$ & n.s. \\
\hline Bebedor en riesgo* & 19,7 & $(14,5-24,9)$ & 34,8 & $(26,5-43,0)$ & 25,2 & $(20,7-29,7)$ & $<0,01$ \\
\hline Bebedor excesivo** & 2,5 & $(0,3-4,6)$ & 10,6 & $(5,2-16,1)$ & 5,5 & $(3,1-7,9)$ & $<0,01$ \\
\hline Con estrés & 20,9 & $(15,6-26,2)$ & 19,1 & $(12,3-26,0)$ & 20,3 & $(16,1-24,4)$ & n.s. \\
\hline
\end{tabular}

n.s: No hay significancia estadística p-valor $>0,05 .{ }^{*}$ Consume 3-5 tragos en una ocasión. ${ }^{*}$ Consume más de 5 tragos en una ocasión. Un trago estándar en Chile son aproximadamente 15,5 g de alcohol absoluto ${ }^{8}$.

ambos parámetros. El 15,6\% de los estudiantes presentaba CT elevado, siendo significativamente mayor en mujeres. El 15,8\% presentaba niveles bajos de cHDL siendo significativamente mayor en varones (Tabla 2). En relación a los quintiles de ingreso, sólo se encontró diferencia significativa en los niveles de colesterol LDL elevado, con un p-valor $<0,05$ (Tabla 3).

\section{Glicemia}

El 2,1\% de los estudiantes presentaba glicemia elevada en ayuno, sin diferencias significativas según género y quintil de ingreso. No se encontraron glicemias $\geq 126 \mathrm{mg} / \mathrm{dL}$ (Tablas 2 y 3 ).

\section{Presión arterial}

El 39,5\% de los estudiantes presentaba PA superior al rango normal; el 35,1\% presentaba PA que podría corresponder a un estado de prehipertensión y 4,4\% presentaba PA que podría corresponder a un estado de HTA, siendo para ambos caso significativamente mayor en varones; no existen diferencias significativas entre PA y quintil de ingreso (Tablas 2 y 3 ).

\section{Síndrome metabólico}

El 4,9\% de los estudiantes presentaron SM, sin diferencias significativas según género y quintil de ingreso (Tablas 2 y 3 ).

\section{Actividad física}

El 87,8\% de los estudiantes era sedentario, siendo significativamente mayor en mujeres (Tabla 2). No se encontraron diferencias significativas según quintil de ingreso (Tabla 3 ).

\section{Consumo de tabaco}

El 28,3\% de los estudiantes era "fumador actual": el 26,2\% era "fumador diario", con un promedio de 4,6 cigarrillos/día y el 2,1\% era "fumador ocasional"; el 13,0\% era "ex-fumador"; sin diferencias significativas según género y quintil de 
Tabla 3. Prevalencias e intervalos de confianza (95\%) de factores de riesgo cardiovascular en estudiantes de la Universidad Austral de Chile, según quintil de ingreso

\begin{tabular}{|c|c|c|c|c|c|c|}
\hline $\begin{array}{l}\text { Factor de riesgo } \\
\text { cardiovascular }\end{array}$ & $\begin{array}{c}1 \\
\%\end{array}$ & $\begin{array}{l}\text { II } \\
\%\end{array}$ & $\begin{array}{c}\text { Quintil } \\
\text { III } \\
\%\end{array}$ & $\begin{array}{l}\text { IV } \\
\%\end{array}$ & $\begin{array}{l}\mathbf{v} \\
\%\end{array}$ & $\begin{array}{c}\text { Total } \\
\%\end{array}$ \\
\hline Sobrepeso ${ }^{a}$ & $\begin{array}{c}22,0 \\
(12,4-31,5)\end{array}$ & $\begin{array}{c}12,9 \\
(3,8-22,1)\end{array}$ & $\begin{array}{c}23,9 \\
(13,3-34,6)\end{array}$ & $\begin{array}{c}25,0 \\
(12,8-37,2)\end{array}$ & $\begin{array}{c}28,9 \\
(20,2-37,7)\end{array}$ & $\begin{array}{c}23,4 \\
(19,0-27,7)\end{array}$ \\
\hline Obesidad ${ }^{a}$ & $\begin{array}{c}3,7 \\
(0,8-10,3)\end{array}$ & $\begin{array}{c}4,8 \\
(1,0-13,5)\end{array}$ & $\begin{array}{c}4,2 \\
(0,9-11,9)\end{array}$ & $\begin{array}{c}5,4 \\
(1,1-14,9)\end{array}$ & $\begin{array}{c}7,0 \\
(1,9-12,1)\end{array}$ & $\begin{array}{c}5,2 \\
(2,8-7,5)\end{array}$ \\
\hline Obesidad abdominal $1^{\text {a }}$ & $\begin{array}{c}7,3 \\
(1,1-13,6)\end{array}$ & $\begin{array}{c}9,7 \\
(1,5-17,8)\end{array}$ & $\begin{array}{c}5,6 \\
(1,6-13,8)\end{array}$ & 0 & $\begin{array}{c}6,1 \\
(1,3-10,9)\end{array}$ & $\begin{array}{c}6,0 \\
(3,5-8,5)\end{array}$ \\
\hline Obesidad abdominal $2^{\mathrm{a}}$ & $\begin{array}{c}22,0 \\
(12,4-31,5)\end{array}$ & $\begin{array}{c}21,0 \\
(10,8-31,9)\end{array}$ & $\begin{array}{c}22,5 \\
(12,1-32,9)\end{array}$ & $\begin{array}{c}32,1 \\
(19,0-45,2)\end{array}$ & $\begin{array}{c}23,7 \\
(15,4-31,9)\end{array}$ & $\begin{array}{c}23,9 \\
(19,5-28,3)\end{array}$ \\
\hline Obesidad abdominal $3^{a}$ & $\begin{array}{c}17,1 \\
(8,3-25,8)\end{array}$ & $\begin{array}{c}16,1 \\
(6,2-26,1)\end{array}$ & $\begin{array}{c}14,1 \\
(5,3-22,9)\end{array}$ & $\begin{array}{c}17,9 \\
(6,9-28,8)\end{array}$ & $\begin{array}{c}15,8 \\
(8,7-22,9)\end{array}$ & $\begin{array}{c}16,1 \\
(12,3-19,9)\end{array}$ \\
\hline Colesterol total elevado & $\begin{array}{c}13,4 \\
(5,4-21,4)\end{array}$ & $\begin{array}{c}16,1 \\
(6,2-26,1)\end{array}$ & $\begin{array}{c}14,1 \\
(5,3-22,9)\end{array}$ & $\begin{array}{c}25,0 \\
(12,8-37,2)\end{array}$ & $\begin{array}{c}13,2 \\
(6,5-19,8)\end{array}$ & $\begin{array}{c}15,6 \\
(11,8-19,3)\end{array}$ \\
\hline Colesterol LDL elevado ${ }^{b}$ & $\begin{array}{c}17,1 \\
(8,3-25,8)\end{array}$ & $\begin{array}{c}21,0 \\
(10,0-31,9)\end{array}$ & $\begin{array}{c}14,1 \\
(5,3-22,9)\end{array}$ & $\begin{array}{c}33,9 \\
(20,6-47,2)\end{array}$ & $\begin{array}{c}14,0 \\
(7,2-20,9)\end{array}$ & $\begin{array}{c}18,7 \\
(14,7-22,7)\end{array}$ \\
\hline Colesterol HDL bajo ${ }^{a}$ & $\begin{array}{c}23,2 \\
(13,4-32,9)\end{array}$ & $\begin{array}{c}17,7 \\
(7,4-28,1)\end{array}$ & $\begin{array}{c}14,1 \\
(5,3-22,9)\end{array}$ & $\begin{array}{c}17,9 \\
(6,9-28,8)\end{array}$ & $\begin{array}{c}9,6 \\
(3,8-15,5)\end{array}$ & $\begin{array}{c}15,8 \\
(12,1-19,6)\end{array}$ \\
\hline Triglicéridos elevados ${ }^{\mathrm{a}}$ & $\begin{array}{c}6,1 \\
(2,0-13,7)\end{array}$ & $\begin{array}{c}4,8 \\
(1,0-13,5)\end{array}$ & $\begin{array}{c}7,0 \\
(2,3-15,7)\end{array}$ & $\begin{array}{c}5,4 \\
(1,1-14,9)\end{array}$ & $\begin{array}{c}5,3 \\
(0,7-9,8)\end{array}$ & $\begin{array}{c}5,7 \\
(3,3-8,2)\end{array}$ \\
\hline Glicemia elevadaa & $\begin{array}{c}1,2 \\
(0,03-6,6)\end{array}$ & $\begin{array}{c}3,2 \\
(0,4-11,2)\end{array}$ & $\begin{array}{c}1,4 \\
(0,03-7,6)\end{array}$ & $\begin{array}{c}3,6 \\
(0,4-12,3)\end{array}$ & $\begin{array}{c}1,8 \\
(0,2-6,2)\end{array}$ & $\begin{array}{c}2,1 \\
(0,5-3,6)\end{array}$ \\
\hline Pre-hipertensión ${ }^{\mathrm{a}}$ & $\begin{array}{c}31,7 \\
(21,0-42,4)\end{array}$ & $\begin{array}{c}35,5 \\
(22,8-48,2)\end{array}$ & $\begin{array}{c}33,8 \\
(22,1-45,5)\end{array}$ & $\begin{array}{c}39,3 \\
(25,6-53,0)\end{array}$ & $\begin{array}{c}36,0 \\
(26,7-45,2)\end{array}$ & $\begin{array}{c}35,1 \\
(30,2-40,0)\end{array}$ \\
\hline Hipertensión ${ }^{a}$ & $\begin{array}{c}4,9 \\
(1,3-12,0)\end{array}$ & $\begin{array}{c}4,8 \\
(1,0-13,5)\end{array}$ & $\begin{array}{c}4,2 \\
(0,9-11,9)\end{array}$ & $\begin{array}{c}1,8 \\
(0,04-9,6)\end{array}$ & $\begin{array}{c}5,3 \\
(0,7-9,8)\end{array}$ & $\begin{array}{c}4,4 \\
(2,2-6,6)\end{array}$ \\
\hline Síndrome metabólico ${ }^{a}$ & $\begin{array}{c}4,9 \\
(1,3-12,0)\end{array}$ & $\begin{array}{c}3,2 \\
(0,4-11,2)\end{array}$ & $\begin{array}{c}7,0 \\
(2,3-15,7)\end{array}$ & $\begin{array}{c}7,1 \\
(2,0-17,3)\end{array}$ & $\begin{array}{c}3,5 \\
(1,0-8,7)\end{array}$ & $\begin{array}{c}4,9 \\
(2,6-7,2)\end{array}$ \\
\hline Sedentarismo ${ }^{a}$ & $\begin{array}{c}90,2 \\
(83,2-97,3)\end{array}$ & $\begin{array}{c}93,5 \\
(84,3-98,2)\end{array}$ & $\begin{array}{c}93,0 \\
(84,3-97,7)\end{array}$ & $\begin{array}{c}80,4 \\
(69,1-91,7)\end{array}$ & $\begin{array}{c}83,3 \\
(76,1-90,6)\end{array}$ & $\begin{array}{c}87,8 \\
(84,4-91,2)\end{array}$ \\
\hline Fumador actuala $^{\mathrm{a}}$ & $\begin{array}{c}20,7 \\
(11,3-30,1)\end{array}$ & $\begin{array}{c}37,1 \\
(24,3-49,9)\end{array}$ & $\begin{array}{c}35,2 \\
(23,4-47,0)\end{array}$ & $\begin{array}{c}17,9 \\
(6,9-28,8)\end{array}$ & $\begin{array}{c}29,8 \\
(21,0-38,7)\end{array}$ & $\begin{array}{c}28,3 \\
(23,7-32,9)\end{array}$ \\
\hline Bebedor en riesgo*a & $\begin{array}{c}13,4 \\
(5,4-21,4)\end{array}$ & $\begin{array}{c}29,0 \\
(16,9-41,1)\end{array}$ & $\begin{array}{c}31,0 \\
(19,5-42,4)\end{array}$ & $\begin{array}{c}25,0 \\
(12,8-37,2)\end{array}$ & $\begin{array}{c}28,1 \\
(19,4-36,8)\end{array}$ & $\begin{array}{c}25,2 \\
(20,7-29,7)\end{array}$ \\
\hline Bebedor excesivo**a & $\begin{array}{c}9,8 \\
(2,7-16,8)\end{array}$ & $\begin{array}{c}6,5 \\
(1,8-15,7)\end{array}$ & $\begin{array}{c}2,8 \\
(0,3-9,8)\end{array}$ & $\begin{array}{c}3,6 \\
(0,4-12,3)\end{array}$ & $\begin{array}{c}4,4 \\
(1,4-9,9)\end{array}$ & $\begin{array}{c}5,5 \\
(3,1-7,9)\end{array}$ \\
\hline Con estrés ${ }^{a}$ & $\begin{array}{c}17,1 \\
(8,3-25,8)\end{array}$ & $\begin{array}{c}17,7 \\
(7,4-28,1)\end{array}$ & $\begin{array}{c}23,9 \\
(13,3-34,6)\end{array}$ & $\begin{array}{c}16,1 \\
(5,6-26,6)\end{array}$ & $\begin{array}{c}23,7 \\
(15,4-31,9)\end{array}$ & $\begin{array}{c}20,3 \\
(16,1-24,4)\end{array}$ \\
\hline
\end{tabular}

${ }^{a}$ No hay significancia estadística p-valor $>0,05$. ${ }^{\text {DD }}$ iferencia estadísticamente significativa $p$-valor $<0,05 .{ }^{*}$ Consume $3-5$ tragos en una ocasión. ${ }^{* *}$ Consume más de 5 tragos en una ocasión. Un trago estándar en Chile son aproximadamente 15,5 g de alcohol absoluto ${ }^{8}$.

ingreso (Tabla 2 y 3 ). El promedio de edad en que comenzaron a fumar fue de 15,7 años.

\section{Consumo de alcohol}

El 79,5\% de los estudiantes consumía alcohol; el $48,8 \%$ era bebedor moderado, $25,2 \%$ era bebedor en riesgo y 5,5\% era bebedor excesivo; existiendo diferencias significativas entre cantidad consumida de alcohol en una oportunidad y género (Tabla 2). En relación a la frecuencia de consumo de alcohol, 56,6\% bebía sólo en ocasiones especiales $(60,2 \%$ de las mujeres y $50,4 \%$ de 


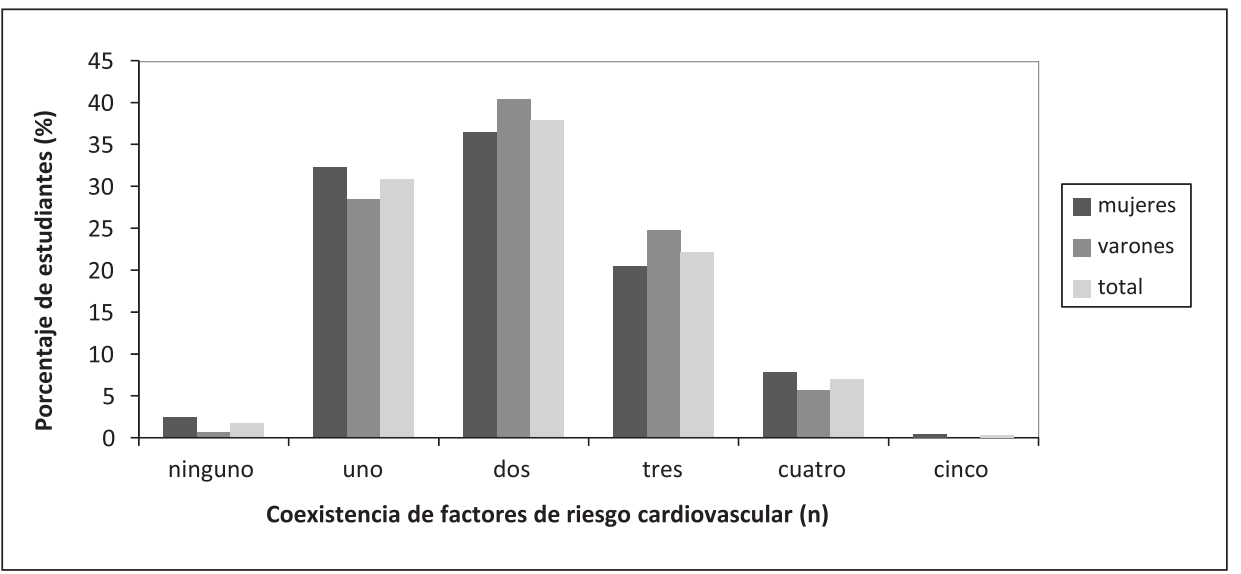

Figura 1. Coexistencia de factores de riesgo cardiovascular en estudiantes de la Universidad Austral de Chile.

Tabla 4. Perfil cardiovascular en estudiantes de la Universidad Austral de Chile

\begin{tabular}{|c|c|c|c|c|c|c|}
\hline & \multicolumn{2}{|c|}{ Total $(n=385)$} & \multicolumn{2}{|c|}{ Mujeres $(n=244)$} & \multicolumn{2}{|c|}{ Varones $(n=141)$} \\
\hline & Media* & DE & Media* & DE & Media* & DE \\
\hline IMC $\left(\mathrm{kg} / \mathrm{m}^{2}\right)$ & 23,8 & 3,2 & 23,9 & 3,2 & 23,7 & 3,3 \\
\hline $\mathrm{CC}(\mathrm{cm})$ & 76,7 & 8,8 & 76,8 & 8,5 & 76,7 & 9,3 \\
\hline $\mathrm{CT}(\mathrm{mg} / \mathrm{dL})$ & 170,1 & 31,9 & 174,2 & 32,3 & 163,1 & 30,0 \\
\hline $\mathrm{cLDL}(\mathrm{mg} / \mathrm{dL})$ & 103,7 & 30,7 & 106,5 & 31,0 & 98,9 & 29,6 \\
\hline $\mathrm{cHDL}(\mathrm{mg} / \mathrm{dL})$ & 49,8 & 10,1 & 51,5 & 9,9 & 46,9 & 9,9 \\
\hline $\mathrm{TG}(\mathrm{mg} / \mathrm{dL})$ & 83,1 & 42,6 & 81,3 & 40,2 & 86,1 & 46,4 \\
\hline Glicemia (mg/dL) & 82,6 & 7,9 & 82,2 & 8,1 & 83,1 & 7,8 \\
\hline PAS (mmHg) & 113,2 & 10,9 & 110,6 & 9,5 & 117,6 & 11,9 \\
\hline PAD $(\mathrm{mmHg})$ & 72,6 & 7,9 & 71,7 & 7,4 & 74,2 & 8,3 \\
\hline
\end{tabular}

IMC: índice de masa corporal. CC: circunferencia cintura. CT: colesterol total. CLDL: colesterol LDL. CHDL: colesterol HDL. TG: triglicéridos. PAS: presión arterial sistólica. PAD: presión arterial diastólica. DE: desviación estándar. No hay significancia estadística p-valor >0,05. *Distribución normal de variables continuas (Prueba de normalidad Shapiro-Wilk).

los varones); siendo significativamente mayor en mujeres $(\mathrm{p}<0,05)$. No se encontraron diferencias significativas entre cantidad consumida de alcohol y quintil de ingreso (Tabla 3$)$.

\section{Estrés}

El 20,3\% de los estudiantes se sientía estresado (3,4\% siempre y $16,9 \%$ casi siempre), sin diferencias significativas según género y quintil de ingreso (Tablas 2 y 3 ).

\section{Coexistencia de FRCV}

El 90,9\% de los estudiantes presentaba entre uno y tres FRCV, sin diferencias significativas entre género $(\mathrm{p}>0,05)$ (Figura 1$)$. La asociación más frecuente en mujeres fue sedentarismo y tabaquismo y en varones sedentarismo y PA elevada.

Se utilizó la media y DE para describir las variables continuas, encontrándose todas dentro de los valores normales, según los puntos de corte utilizados y no se observaron diferencias significativas entre género (Tabla 4).

\section{Discusión}

El objetivo del trabajo fue reconocer precozmente FRCV modificables en los jóvenes universitarios, siendo el sedentarismo, PA y consumo de alcohol elevados los de mayor prevalencia. No 
obstante, en los últimos años se han investigado los "factores de riesgo emergentes o no tradicionales"19,20, que pueden aparecer en personas que no presentan los factores de riesgo tradicionales, pero que al no ser considerados en este estudio, el riesgo cardiovascular de esta muestra podría estar subestimado.

El exceso de peso en los estudiantes es levemente superior a lo publicado en la Encuesta Nacional de Salud (ENS 2003) en jóvenes ${ }^{3}$, pero inferior a lo encontrado en la ENS 2009-2010 ${ }^{8} \mathrm{y}$ en otros estudios en jóvenes ${ }^{6,7}$; hecho atribuible a que el sobrepeso y obesidad aumentan con la edad y este estudio incorporó estudiantes con una edad promedio de 19 años, lo que podría constituir una posible limitación.

Considerando que la obesidad constituye un factor de riesgo condicionante no sólo de las ECV, sino también de la mayoría de las enfermedades crónicas $^{12}$, es importante tomar medidas de prevención y control del sobrepeso y obesidad.

La obesidad abdominal, medida a través de CC es un buen indicador de riesgo cardiovascular ${ }^{21,22}$, por lo que su detección temprana cobra especial interés, debido a que aumenta con la edad ${ }^{23}$. Este trabajo determinó la prevalencia de obesidad abdominal usando tres criterios con diferentes puntos de corte. Al usar el criterio 1 se encontró baja prevalencia, en relación a otros estudios nacionales, utilizando el mismo criterio ${ }^{6,7}$. La mayor prevalencia de obesidad abdominal se encontró al usar el criterio 2, que es más estricto que el anterior, siendo mayor en mujeres, lo que coincide con los resultados de la ENS $2003^{3}$. Al usar el criterio 3 , el cual representa mejor a la población chilena y permite comparaciones entre estudios nacionales ${ }^{3,15}$, la prevalencia de obesidad abdominal fue inferior a lo informado en la ENS 2009-2010, donde usaron el mismo punto de corte . $^{8}$

Aunque la naturaleza de este estudio no permite hacer un diagnóstico confirmatorio de HTA, es preocupante que el $35,1 \%$ de los estudiantes presente pre-hipertensión, debido a las altas probabilidades de desarrollar HTA en un futuro próximo $^{24}$. La HTA es uno de los mayores FRCV modificable, por lo tanto, es necesario prevenir su aparición desde la juventud. La prevalencia $(4,4 \%)$ de HTA, concuerda con estudios realizados en otras universidades ${ }^{5,25}$, y en adultos jóvenes ${ }^{6}$, pero es levemente superior a lo encontrado en la ENS 2009-2010.
En relación a las dislipidemias, si se comparan estos resultados con los reportados en jóvenes a nivel nacional en la ENS 2003 y la ENS 2009-20103,8, se observan mayores prevalencias de hipercolesterolemia y de cLDL elevado; en cambio los TG aumentados y cHDL bajo presentan prevalencias inferiores. Al comparar con otros estudios similares $^{5-7}$, la prevalencia de cLDL es semejante; sin embargo, la hipercolesterolemia, TG aumentados y cHDL bajo presentan prevalencias inferiores. En el presente estudio se destaca la mayor prevalencia de hipercolesterolemia en mujeres, situación distinta a las reportadas ${ }^{3,5,7,8}$, donde no existen diferencias entre los géneros.

La glicemia elevada en ayuno aumenta el riesgo de desarrollar diabetes a futuro ${ }^{26}$. Dos coma uno porciento de los estudiantes presentó valores elevados de glicemia en ayuno, pero sin sobrepasar los $110 \mathrm{mg} / \mathrm{dL}$. Para descartar posible intolerancia a la glucosa sería recomendable evaluar además los niveles de glicemia a $2 \mathrm{~h}$ de sobrecarga de glu$\cos ^{27}$. Nuestros resultados concuerdan con otros estudios realizados en jóvenes, donde $1,3 \%$ y $1,4 \%$ presentaron hiperglicemia ${ }^{6,7}$. No se encontraron casos de posible diabetes, lo que no es extraño, debido a que esta enfermedad aparece en edades más avanzadas ${ }^{3}$.

El SM es un FRCV mayor e independiente, cuya presencia no sólo aumenta el riesgo de desarrollar DM2 y ECV ${ }^{28}$, sino también otras enfermedades crónicas $^{29}$, por lo que su diagnóstico precoz es importante. La prevalencia de SM en nuestros estudiantes fue lo esperado para una población menor a 25 años, siendo semejante a lo encontrado en la ENS $2003^{3}$ e inferior a lo reportado por la ENS $2009-2010^{8}$, esto último se podría explicar porque en este estudio se usó un mayor umbral para glicemia elevada.

El sedentarismo fue el FRCV que presentó mayor prevalencia, alcanzando 87,8\%; coincidiendo con otros estudios nacionales, que utilizaron el mismo criterio para su evaluación ${ }^{3,10}$. El sedentarismo es un factor de riesgo modificable, no sólo de ECV, sino además de otras enfermedades crónicas $^{30,31}$; por lo tanto, es importante revertir esta situación a través de políticas públicas que promuevan la actividad física como una forma de vida.

La prevalencia de tabaquismo es menor que la señalada por estudios desarrollados en universitarios y población joven en general ${ }^{3,5,7,8,32,33}$; esto 
Factores de riesgo cardiovascular en estudiantes universitarios - M. A. Martínez et al

podría atribuirse a una limitación del cuestionario que no consideró el tiempo que los ex-fumadores habían dejado de fumar; por lo que el 13\% de exfumadores podría estar en la categoría de fumador. Además, este hábito tiende a aumentar con los años de universidad ${ }^{25,34}$.

El hábito de consumo de alcohol en los estudiantes, concuerda con lo encontrado en educación superior a nivel nacional ${ }^{35} \mathrm{y}$ lo publicado en la ENS 2009-20108, donde la XIV Región de los Ríos, en la que se inserta la UACh, registra el mayor consumo de alcohol en el país. Al comparar estos resultados con un estudio realizado en una universidad española, se observa una prevalencia $\operatorname{similar}^{36}$. Coincidente con otros estudios nacionales $^{8,35}$, el consumo de alcohol se concentra preferentemente en un día a la semana, siendo los varones los que más beben. En relación a la intensidad de consumo de alcohol y observando la tendencia encontrada en estudiantes de los últimos años escolares ${ }^{37}$, se esperaría que en los próximos años el consumo excesivo vaya en aumento.

El estrés es un FRCV emergente cuyo efecto es comparable con HTA e hipercolesterolemia ${ }^{38-40}$. El 20,3\% de los estudiantes señaló sentirse estresado, cifra similar a lo encontrado a nivel nacional en jóvenes ${ }^{41}$. El estrés es un factor de riesgo frecuente en estudiantes universitarios ${ }^{42,43}$ y una limitación del presente estudio es no haber caracterizado mejor este parámetro.

A pesar que la mayor proporción de la muestra pertenece a los 3 quintiles más pobres, llama la atención que no se encontró asociación con los FRCV analizados, ya que existen estudios que relacionan la mayor prevalencia de estos factores de riesgo en aquellos estratos socioeconómicos más bajos ${ }^{44,45}$.

Los FRCV tienden a agruparse en una misma persona, siendo frecuente la coexistencia de dos o más FRCV, aumentando el riesgo de ECV a medida que éstos se suman. Más de la mitad de los estudiantes presenta dos o más FRCV, aumentando considerablemente las probabilidades de sufrir ECV en el futuro.

Esta investigación evidenció que los estudiantes que ingresan a la UACh poseen estilos de vida no saludables asociados a los principales FRCV. Si bien el estudio presenta ciertas limitaciones, entre las que destacan el uso de cuestionarios simplificados en la evaluación de los estilos de vida, así como el no haber incluido estudiantes de cursos superiores, para aumentar el rango de edad de la muestra, consideramos que los resultados concuerdan con estudios a nivel nacional y reflejan la realidad de la juventud de nuestro país.

Estos resultados entregan información relevante para desarrollar políticas internas orientadas a la prevención y control de FRCV en nuestros estudiantes. En este sentido, se visualiza como necesidad fundamental, la incorporación en el currículo universitario de contenidos transversales que propicien la adopción de estilos de vida saludables, con el fin de prevenir a futuro la incidencia de ECV. Por ejemplo, promoción de una alimentación saludable, desincentivo del consumo de tabaco y alcohol, aumento de la oferta de programas asociados al currículo que promuevan la práctica regular de actividad física.

\section{Referencias}

1. WHO. World Health Organization. The World Health Report 2002. Reducing Risks, Promoting Healthy Life. WHO 2002. Disponible en: http://www.who.int/ whr/2002/en/whr02_en.pdf [Consultado el 31 de enero de 2011].

2. O'Donnell CJ, Elosua R. Factores de riesgo cardiovascular. Perspectivas derivadas del Framingham Heart Study. Rev Esp Cardiol 2008; 61: 299-310.

3. MINSAL, Ministerio de Salud, Gobierno de Chile. Primera Encuesta Nacional de Salud. Chile 2003. Disponible en: http://epi.minsal.cl/epi/html/invest/ENS/ InformeFinalENS.pdf [Consultada el 20 de enero de 2011].

4. INE. Instituto Nacional de Estadísticas de Chile. Estadísticas vitales, informe anual 2008. Diciembre 2010. Disponible en: http://www.ine.cl/canales/menu/publicaciones/calendario_de_publicaciones/pdf/21_12_10/ vit_08211210.pdf [Consultado el 29 de marzo de 2011].

5. Chiang MT, Casanueva V, Cid X, González U, Olate P, Nickel F, et al. Factores de riesgo cardiovascular en estudiantes universitarios chilenos. Salud Pública de México 1999; 41: 444-51.

6. Bustos P, Amigo H, Arteaga A, Acosta AM, Rona RJ. Factores de Riesgo de Enfermedad Cardiovascular en Adultos Jóvenes. Rev Med Chile 2003; 131: 973-80.

7. Palomo I, Torres G, Alarcón M, Maragaño P, Leiva E, Mujica V. Alta prevalencia de factores de riesgo cardiovascular clásicos en una población de estudiantes universitarios de la región centro-sur de Chile. Rev Esp Cardiol 2006; 59: 1099-105.

8. MINSAL. Ministerio de Salud. Gobierno de Chile. En- 
Factores de riesgo cardiovascular en estudiantes universitarios - M. A. Martínez et al

cuesta Nacional de Salud ENS Chile 2009-2010. Chile 2011. Disponible en: http://www.redsalud.gov.cl/portal/ url/item/99c12b89738d80d5e04001011e0113f8.pdf [Consultado el 31 de enero 2011].

9. Ministerio de Planificación (MIDEPLAN). Gobierno de Chile. Encuesta Nacional de Caracterización Socioeconómica CASEN 2003. Límites mínimos y máximos del ingreso autónomo per-cápita que definen cada uno de los quintiles por quintil de ingreso autónomo del hogar regional según región, zona y quintil. http:// www.mideplan.gob.cl/casen/Estadisticas/ingresos.html [Consultado el 6 de septiembre 2011].

10. MINSAL, Ministerio de Salud, Gobierno de Chile. Departamento de Epidemiología. Departamento de Promoción de Salud. II Encuesta Nacional de Calidad de Vida y Salud. Chile 2006. Cuestionario. Disponible en: http://epi.minsal.cl/epi/html/sdesalud/calidaddevida2006/cuestionario.pdf [Consultado el 31 de enero 2011].

11. OPS, Organización Panamericana de la Salud. Herramienta para Vigilancia de ENTs: Factores de Riesgo para Enfermedades No Transmisibles. División de Prevención y Control de Enfermedades, Washignton DC 20037, USA. http://www.paho.org/spanish/ad/dpc/nc/ncd-survtools.htm [Consultado el 31 de enero 2011].

12. WHO. World Health Organization. Obesity: Preventing and Managing The Global Epidemic. WHO TechnicalReport Series 894. Geneva 2000. Disponible en: http:// whqlibdoc.who.int/trs/who_trs_894.pdf [Consultado el 31 de enero de 2011].

13. MINSAL. Ministerio de Salud. Gobierno de Chile. Guía Clínica Hipertensión Arterial Primaria o Esencial en Personas de 15 años y más.1st Ed. Santiago. Chile 2005. Disponible en: http://www.sld.cu/galerias/pdf/servicios/ hta/guia_hipertension_arterial_chile_2005.pdf [Consultado el 20 de enero 2011].

14. Friedewald WT, Levy RI, Fredrickson DS. Estimation of the Concentration of Low-Density Lipoprotein Cholesterol in Plasma, Without Use of the Preparative Ultracentrifuge. Clinical Chemistry 1972; 18: 499-502.

15. Arteaga AA. Nuevos indicadores de Riesgo Cardiovascular. Rev Chil Cardiol 2009; 28: 381-4.

16. Third Report of the National Cholesterol Education Program (NCEP) Expert Panel on Detection, Evaluation, and Treatment of High Blood Cholesterol in Adults (Adult Treatment Panel III) Final Report (2002). Circulation 2002; 106: 3143-421.

17. Chobanian AV, Bakris GL, Black HR, Cushman WC, Green LA, Izzo, et al. Seventh Report of the Joint National Committee on Prevention, Detection, Evaluation, and Treatment of High Blood Pressure. Hypertension
2003; 42: 1206-52.

18. MINSAL. Ministerio de Salud. Gobierno de Chile. Estrategia Nacional sobre Alcohol: Reducción del consumo de riesgo y sus consecuencias sociales y sanitarias. Propuesta de un desafío bicentenario para Chile. Chile 2010. Disponible en: http://www.redsalud.gov.cl/portal/ url/item/952e1e6b068b7eb6e04001011e0126c7.pdf [Consultado el 31 de enero 2011].

19. Acevedo M, Tagle R, Simpfendorfer C. [Non-traditional risk factors for Atherosclerosis]. Rev Med Chile 2001; 129: 1212-21.

20. Hackam DG, Anand SS. Emerging Risk Factors for Atherosclerotic Vascular Disease: A Critical Review of the Evidence. JAMA. 2003; 290: 932-40.

21. Han TS, van Leer EM, Seidell JC, Lean ME. Waist circumference action levels in the identification of cardiovascular risk factors: prevalence study in a random sample. BMJ 1995; 31: 1401-5.

22. Gustat J, Elkasabany A, Srinivasan S, Berenson GS. Relation of Abdominal Height to Cardiovascular Risk Factors in Young Adults. The Bogalusa Heart Study. Am J Epidemiol 2000; 151: 885-91.

23. Durán S, Castillo M, Vio F. Diferencias en la calidad de vida de estudiantes universitarios de diferente año de ingreso del Campus Antumapu. Rev Chil Nutr 2009; 36: 200-9.

24. Grupo de Trabajo para el Tratamiento de la Hipertensión Arterial de la Sociedad Europea de Hipertensión (ESH) y de la Sociedad Europea de Cardiología (ESC). Guías de práctica clínica para el tratamiento de la hipertensión arterial. Rev Esp Cardiol 2007; 60: 968. e1-e94.

25. Mc Coll P, Amador M, Aros J, Lastra A, Pizarro C. Prevalencia de factores de riesgo de enfermedades crónicas no transmisibles en estudiantes de medicina de la Universidad de Valparaíso. Rev Chil Pediatr 2002; 73: 478-82.

26. American Diabetes Association. Standards of Medical Care in Diabetes-2008. Diabetes Care 2008; 31 (Suppl 1): S12-S54. Disponible en: http://care.diabetesjournals. org/content/31/Supplement_1/S12.full.pdf+html [Consultado el 31 de enero 2011].

27. WHO. World Health Organization. Definition and Diagnosis of Diabetes Mellitus and Intermediate Hyperglycemia: Report of a WHO/IDF Consultation. 2006. Disponible en: http://whqlibdoc.who.int/publications/2006/9241594934_eng.pdf [Consultado el 16 de noviembre de 2011].

28. Grundy SM, Cleeman JI, Daniels SR, Donato KA, Eckel RH, Franklin BA, et al. Diagnosis and Management of the Metabolic Syndrome: An American Heart Association/National Heart, Lung, and Blood Institute Scientific Statement. Circulation 2005; 112: 285-90. 
Factores de riesgo cardiovascular en estudiantes universitarios - M. A. Martínez et al

29. von Bernhardi R, Zanlungo S, Arrese M, Arteaga A, Rigotti A. El síndrome metabólico: De factor agravante a principal factor de riesgo patogénico en diversas enfermedades crónicas. Rev Med Chile 2010; 138: 1012-9.

30. Warburton DE, Nicol CW y Bredin SS. Health benefits of physical activity: the evidence. Can Med Assoc J 2006; 174: 801-9.

31. Proper KI, Singh AS, van Mechelen W, Chinapaw MJM. Sedentary Behaviors and Health Outcomes Among Adults. A Systematic Review of Prospective Studies. Am J Prev Med 2011; 40: 174-82.

32. CONACE. Consejo Nacional para el Control de Estupefacientes. Octavo Estudio Nacional de Drogas en Población General de Chile, 2008. Área Evaluación y Estudios. Junio 2009. Disponible en: http://bibliodrogas.cl/ biblioteca/digital/resumen_informe_VIII_estudio_drogas_poblaciongeneral_junio2009.pdf [Consultado el 31 de enero 2011].

33. Sotomayor H, Behn V, Cruz M, Naveas R, Sotomayor C, Fernández P. et al. Tabaquismo en académicos, no académicos y estudiantes de la Universidad de Concepción. Rev Med Chile 2000; 128: 977-84.

34. Halperin AC, Smith SS, Heiligenstein E, Brown D, Fleming MF. Cigarette smoking and associated health risks among students at five universities. Nicotine \& Tobacco Research 2010; 12: 96-104.

35. CONACE, Consejo Nacional para el Control de Estupefacientes. Informe Sobre Uso de Drogas en Estudiantes de la Educación Superior. Estudio nacional de drogas en la población general de Chile, 2002. Junio 2003. Disponible en: http://intranet.injuv.gob.cl/cedoc/CONACE/ Uso_drogas_estudiantes_educacion_superior_CONACE_2004.pdf [Consultado el 31 de enero 2011].

36. Tirado R, Aguaded JI, Marín I. Factores de protección y de riesgo del consumo de alcohol en alumnos de la universidad de Huelva. Salud y Drogas 2009; 9: 165-83.

37. CONACE, Consejo Nacional para el Control de Estupefacientes. Octavo Estudio Nacional de Drogas en Población Escolar de Chile 2009. Área de Evaluación y Estudios. Septiembre 2010. Principales resultados. Disponible en: http://www.conacedrogas.cl/portal/index. php? option $=$ com_content $\&$ view $=$ article $\&$ id $=282 \&$ Ite mid=368 [Consultado el 18 de marzo 2011].

38. Rozanski A, Blumenthal JA, Kaplan J. Impact of Psychological Factors on the Pathogenesis of Cardiovascular Disease and Implications for Therapy. Circulation 1999; 99: 2192-217.

39. Bunker SJ, Colquhoun DM, Esler MD, Hickie IB, Hunt D, Jelinek VM, et al. "Stress" and coronary heart disease: psychosocial risk factors. MJA 2003; 178: 272-6.

40. Rosengren A, Hawken S, Ounpuu S, Sliwa K, Zubaid M, Almahmeed WA, et al. For the INTERHEART investigators. "Association of psychosocial risk factors with risk of acute myocardial infarction in 11,119 cases and 13,648 controls from 52 countries. (the INTERHEART study): case-control study". Lancet 2004; 364: 953-62.

41. MINSAL, Ministerio de Salud, Gobierno de Chile. Departamento de Epidemiología. Departamento de Promoción de Salud. II Encuesta Nacional de Calidad de Vida y Salud. Chile. 2006. Informe de resultados total nacional. Disponible en: http://epi.minsal.cl/epi/html/ sdesalud/calidaddevida2006/Informe $\% 20$ Final $\% 20$ Encuesta\%20de\%20Calidad\%20de\%20Vida\%20y\%20 Salud\%202006.pdf [Consultado el 31 de enero 2011].

42. Martín IM. Estrés académico en estudiantes universitarios. Apuntes de Psicología 2007; 25: 87-99.

43. Cova F, Alvial W, Aro M, Bonifetti A, Hernández M, Rodríguez C. Problemas de Salud Mental en Estudiantes de la Universidad de Concepción. Terapia Psicológica 2007; 25: 105-12.

44. Jadue L, Vega J, Escobar MC, Delgado I, Garrido C, Lastra P, Espejo F, et al. Factores de riesgo para las enfermedades no transmisibles: Metodología y resultados globales de la encuesta de base del programa CARMEN (Conjunto de Acciones para la Reducción Multifactorial de las Enfermedades no Transmisibles). Rev Med Chile 1999; 127: 1004-13.

45. Koch E, Romero T, Manríquez L, Paredes M, Ortúzar E, Taylor A, et al. Desigualdad educacional y socioeconómica como determinante de mortalidad en Chile: análisis de sobrevida en la cohorte del proyecto San Francisco. Rev Med Chile 2007; 135: 1370-9. 